\title{
Inhibition of circular RNA_0000285 prevents cell proliferation and induces apoptosis in thyroid cancer by sponging microRNA-654-3p
}

\author{
RONGJUN GAO, HUI YE, QINGJUN GAO, NANPENG WANG, YAN ZHOU and HAISONG DUAN
}

Department of Thyroid Surgery, Affiliated Hospital of Guizhou Medical University, Guiyang, Guizhou 550004, P.R. China

Received January 18, 2021; Accepted May 25, 2021

DOI: $10.3892 / 01.2021 .12934$

\begin{abstract}
Thyroid cancer is derived from follicular or thyroid cells and has become the most prevalent malignant tumor of endocrine organs, with increased morbidity and mortality. Circular RNAs (circRNAs) are used as prognostic and predictive markers for different types of cancer. However, the role of circRNA_0000285 in thyroid cancer and its potential molecular mechanism remain unclear. The present study aimed to investigate the roles and underlying molecular mechanism of circRNA_0000285 in thyroid cancer to identify novel treatments for this disease. The target binding site of circRNA_0000285 and microRNA-654-3p (miR-654-3p) were predicted and confirmed via the dual-luciferase reporter and RNA immunoprecipitation (RIP) assays. Thyroid cancer cell viability and apoptosis were determined via the MTT assay and flow cytometric analysis, respectively, whereas the expression levels of circRNA_0000285 and miR-654-3p were determined via reverse transcription-quantitative PCR analysis. In addition, the protein expression levels of the apoptosis-associated proteins, Bax and B-cell lymphoma 2 (Bcl-2), were detected via western blotting. The results of the dual-luciferase reporter and RIP assays demonstrated that miR-654-3p directly targeted circRNA_0000285. The expression levels of circRNA_0000285 and miR-654-3p in thyroid cancer cells (TPC-1 and FTC133) were upregulated and downregulated, respectively. Knockdown of circRNA_0000285 via small interfering (si)RNA inhibited circRNA_0000285 levels and increased miR-654-3p levels. In addition, miR-654-3p expression decreased following transfection with miR-654-3p inhibitor. Functional experiments demonstrated that circRNA_0000285-siRNA decreased thyroid cancer cell proliferation, promoted cell apoptosis, enhanced Bax expression and suppressed Bcl-2 expression. All these effects were
\end{abstract}

Correspondence to: Dr Hui Ye, Department of Thyroid Surgery, Affiliated Hospital of Guizhou Medical University, 128 Guiyi Street, Yunyan, Guiyang, Guizhou 550004, P.R. China

E-mail: yehui1426118@163.com

Key words: thyroid cancer, circular RNA_0000285, microRNA-654-3p reversed following transfection with miR-654-3p inhibitor. Taken together, the results of the present study suggest that circRNA_0000285 plays a vital role in thyroid cancer progression by regulating miR-654-3p, which provides a potential therapeutic target for this disease.

\section{Introduction}

Thyroid cancer is the most common thyroid malignancy, accounting for $2.6 \%$ of all malignant tumors (1). The majority of thyroid cancer types originate from follicular epithelial cells, and are mainly divided into papillary carcinoma and follicular adenocarcinoma, according to their pathological subtypes (2). Available treatment methods exist, such as surgical treatment (3), thyroid hormone suppression therapy (4) and radioiodine ablation (5) that may effectively inhibit tumors. However, secondary recurrence is common (6). Thus, it is important to identify effective biomarkers and targets to improve the survival rate of patients with thyroid cancer.

Circular RNAs (circRNAs) are non-coding RNAs that play important roles in the development of several diseases, such as cardiovascular disease, nervous system disease and cancer, as well as regulate specific cellular functions, including development, proliferation and invasion (7,8). Lee et al (7) demonstrated the roles of circRNAs in the development of human diseases. Furthermore, Ma et al (8) revealed the potential regulatory role of circRNA-000284 in cervical cancer cell proliferation and invasion by targeting microRNA (miRNA/miR)-506. Previous studies have reported that circRNAs contain several miRNA binding sites, which are involved in the regulation of gene expressions by sponging miRNAs $(9,10)$. circRNA_0000285 has been identified as a novel circRNA, which promotes the metastasis and invasion of several types of cancer by targeting specific miRNAs (11). However, the roles and underlying molecular mechanism of circRNA_0000285 in thyroid cancer remain unclear.

miRNAs are a class of endogenous small RNAs with vital regulatory roles in cells (12). It has been reported that miRNAs are promising markers of several biological processes, including cell proliferation, migration and invasion (13). For example, miR-211 triggers an autophagy-dependent apoptotic response in cervical cancer cells by regulating B-cell lymphoma 2 (Bcl-2) (14). Furthermore, miR-654-3p is a key regulator of cell proliferation and migration that promotes 
the induction of gene reprogramming (15). miR-654-3p is a tumor inhibitory factor that is downregulated in hepatocellular carcinoma (16). Pu et al (17) highlighted that miR-654-3p suppresses non-small cell lung cancer tumorigenesis by inhibiting polo like kinase 4. However, whether miR-654-3p is associated with the development of thyroid cancer remains unknown. Notably, a previous study revealed the interaction sites between circRNA_0000285 and miR-654-3p, and circRNA_0000285 functions as a sponge of miR-654-3p in diabetic nephropathy (18). However, the association between circRNA_0000285 and miR-654-3p in thyroid cancer has not been studied till now.

The present study aimed to investigate the roles and underlying molecular mechanism of circRNA_0000285, and determine the association between circRNA_0000285 and miR-654-3p in thyroid cancer to identify novel strategies for thyroid cancer treatment.

\section{Materials and methods}

Cell culture. The cell lines, TPC-1, FTC133 and Nthy-ori 3-1, were purchased from the American Type Culture Collection and maintained in RPMI-1640 medium (Gibco; Thermo Fisher Scientific, Inc.) supplemented with $10 \%$ fetal bovine serum (HyClone; Cytiva) and $1 \%$ penicillin-streptomycin (Gibco; Thermo Fisher Scientific, Inc.), at $37^{\circ} \mathrm{C}$ with $5 \% \mathrm{CO}_{2}$.

Dual-luciferase reporter assay. StarBase version 2.0 (http://starbase.sysu.edu.cn/index.php) was used to predict the binding sites between circRNA_0000285 and miR-654-3p. The dual-luciferase reporter assay was performed to confirm the binding sites between circRNA_0000285 and miR-654-3p. The wild-type 3'-untranslated region (UTR) of circRNA_0000285 (WT circ_0000285: 5'-GGUGAUGCUUUUCAGCAGACAUU-3'), which contains the miR-654-3p binding site or the mutated target site (MUT circ_0000285: 5'-GGUGAUGCUUUUCAG GUCUGUAU-3') was generated by PCR (using a Transcriptor First Strand cDNA Synthesis kit (cat. no. 04896866001; Roche Molecular Diagnostics) (incubation for $5 \mathrm{~min}$ at $25^{\circ} \mathrm{C}$ followed by $60 \mathrm{~min}$ at $42^{\circ} \mathrm{C}$ ) from total RNA preps extracted from TPC-1 cells (electrophoresis analysis, 1\% agarose gel; visualisation method, ethidium bromide) and cloned into the pMIR vectors (Ambion; Thermo Fisher Scientific, Inc.) to construct the reporter vector circRNA_0000285 wild-type or the circRNA_0000285 mutant-type. 293T cells (American Type Culture Collection) were transfected with circRNA_0000285 wild-type or the mutant sequence, and with miR-654-3p mimic (forward, 5'-UGGUGG GCCGCAGAACAUGUGC-3' and reverse, 5'-ACAUGUUCU GCGGCCCACGAAU-3') or mimic control (forward, 5'-UUC UCCGAACGUGUCACGUUU-3' and reverse, 5'-ACGUGA CACGUUCGGAGAAUU-3'; both purchased from Shanghai GenePharma, Co., Ltd.) using Lipofectamine ${ }^{\circledR} 2000$ (Invitrogen; Thermo Fisher Scientific, Inc.) at $37^{\circ} \mathrm{C}$ for $48 \mathrm{~h}$, according to the manufacturer's instruction. Following cell transfection, $48 \mathrm{~h}$ later the Dual-Luciferase Reporter Assay system (Promega Corporation) was applied to measure luciferase activity and Renilla luciferase was used as a normalization control.

RNA pull-down assay. The pull-down assay was performed as previously described (19). Briefly, the biotinylated
circRNA_0000285 probe and oligo probe (Shanghai GenePharma Co., Ltd.) were incubated with M-280 Streptavidin magnetic beads (cat. no. 60210; Invitrogen; Thermo Fisher Scientific, Inc.) according to the manufacturer's instruction for $2 \mathrm{~h}$ at room temperature to generate probe-coated beads. Subsequently, the biotinylated RNA coated beads were separated with a magnet for 3 min and washed with $1 \mathrm{X}$ B\&W Buffer [2X B\&W Buffer: 10 mM Tris- $\mathrm{HCl}$ (pH 7.5) 1 mM EDTA $2 \mathrm{M} \mathrm{NaCl}$ ].

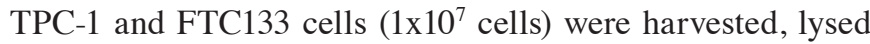
in $500 \mu \mathrm{l}$ lysis buffer (Beyotime Institute of Biotechnology), sonicated and incubated with probe-coated beads overnight at $4^{\circ} \mathrm{C}$. After washing with $1 \mathrm{X} \mathrm{B} \& W$ Buffer, the RNA complexes bound to the beads were eluted and extracted using the RNA isolation kit (Thermo Fisher Scientific, Inc.), and analyzed via reverse transcription-quantitative (RT-q)PCR analysis.

The pull-down efficiency was verified in TPC-1 and FTC133 cells transfected with circRNA_0000285 (human circRNA_0000285 cDNA was synthesized and cloned into the plenti-ciR-GFP-T2A vector) or vector (plenti-ciR-GFP-T2A vector; IGE Biotech Co., Ltd.) using Lipofectamine RNAiMax $^{\circledR}$ (Thermo Fisher Scientific, Inc.) according to the manufacturer's instruction.

RNA immunoprecipitation (RIP) assay. The RIP assay is a powerful tool used to assess the binding of RNA molecules to proteins in cells and to assess the dynamic process of post transcriptional regulatory networks (20). Thyroid cancer cells were transfected with miR-654-3p mimic and mimic control at $37^{\circ} \mathrm{C}$ for $48 \mathrm{~h}$, and were lysed using RIP buffer (Beyotime Institute of Biotechnology). The magnetic beads were coated with anti-Ago2 (1:50; cat. no. ab186733; Abcam) or IgG (1:300; cat. no. ab109489; Abcam) antibodies and the cell lysate was incubated with the magnetic beads at $4^{\circ} \mathrm{C}$ for $1 \mathrm{~h}$, according to the manufacturer's instructions.

$R T$ - $q P C R$. RNA was extracted from thyroid cancer cells and normal thyroid cells using the RNA isolation kit (Thermo Fisher Scientific, Inc.), according to the manufacturer's instructions. cDNA was synthesized using the PrimeScript RT kit (Takara Bio, Inc.) and amplified via qPCR on an ABI PRISM 7900 sequence detection system (Applied Biosystems; Thermo Fisher Scientific, Inc.), with SYBR Premix Ex-Taq (Takara Bio, Inc.). The thermocycling conditions were as follows: Initial denaturation at $95^{\circ} \mathrm{C}$ for 5 min, followed by 38 cycles of denaturation at $95^{\circ} \mathrm{C}$ for $15 \mathrm{sec}$ and annealing/elongation at $60^{\circ} \mathrm{C}$ for $30 \mathrm{sec}$. The following primer sequences (Sangon Biotech Co., Ltd.) were used for qPCR: U6 forward, 5'-CTCGCTTCGGCAGCA CA-3' and reverse, 5'-AACGCTTCACGAATTTGCGT-3'; GAPDH forward, 5'-TCA ACGACCACTTTGTCAAGC TCA-3' and reverse, 5'-GCTGGTGGTCCAGGGGTCTTA CT-3'; miR-654-3p forward, 5'-GGGATGTCTGCTGAC CA-3' and reverse, 5'-CAGTGCGTGTCGTGGA-3'; and circRNA_0000285 forward, 5'-TACCTCTGCAGGCAGG AACT-3' and reverse, 5'-TCACATGAATTTAGGTGGGAC TT-3'. Relative expression levels were calculated using the $2^{-\Delta \Delta \mathrm{Cq}}$ method (21).

Cell transfection. Control-small interfering (si)RNA (1 $\mu \mathrm{g}, 5^{\prime}$ - AAG ACA UUG UGUGUC CGC CT T-3'), 
circRNA_0000285-siRNA $(1 \mu \mathrm{g}, 5$ '-CCCCAGCUAUUCAAG UGUAAA-3'), inhibitor control (50 nM, 5'-AAGUCAGGU GAUGGACAGCAUA-3') and miR-654-3p inhibitor (50 nM, 5'-AAGGUGAUGGUCAGCAGACAUA-3') (all purchased from Shanghai GenePharma Co., Ltd.) were transfected into TPC-1 and FTC133 cells using Lipofectamine $2000^{\circledR}$ (Thermo Fisher Scientific, Inc.) at $37^{\circ} \mathrm{C}$ for $48 \mathrm{~h}$, according to the manufacturer's instructions. After 48 h, RT-qPCR analysis was performed to assess the efficiency of transfection.

MTT assay. Following transfection, TPC-1 and FTC133 cells were cultured for $48 \mathrm{~h}$ and seeded into 96 -well plates. The plates were incubated for 24,48 and $72 \mathrm{~h}$ at $37^{\circ} \mathrm{C}$. Subsequently, cells were treated with $10 \mu \mathrm{l}$ MTT $(5 \mathrm{mg} / \mathrm{ml})$ solution and incubated for an additional $4 \mathrm{~h}$. Following treatment, the solution was removed and $100 \mu$ 1 DMSO was added to each well to dissolve the formazan product. Samples were vibrated using a Microplate Shaker (Thermo Fisher Scientific, Inc.) and optical density was measured at a wavelength of $570 \mathrm{~nm}$, using a multifunctional plate reader (BioTek Instruments, Inc.), according to the manufacturer's instructions.

Flow cytometry. Following incubation of transfected TPC-1 and FTC133 cells for $48 \mathrm{~h}$, the induction of apoptosis was measured using the Annexin V-fluorescein isothiocyanate/PI apoptosis detection kit (BD Biosciences), according to the manufacturer's instructions. Flow cytometry was performed to detect cell apoptosis (early apoptotic cells + late apoptotic cells; upper right quadrant + lower right quadrant) and the results were analyzed using Kaluza Analysis (version 2.1.1.20653; Beckman Coulter, Inc.).

Western blotting. Following transfection, TPC-1 and FTC133 cells were incubated for $48 \mathrm{~h}$ and total proteins were extracted using RIPA buffer (Invitrogen; Thermo Fisher Scientific, Inc.). BCA assay (Thermo Fisher Scientific, Inc.) was used to measure the protein concentrations. Equal amounts of proteins (40 $\mu \mathrm{g} /$ lane) were separated via $12 \%$ SDS-PAGE, transferred onto PVDF membranes and blocked with 5\% skimmed milk in PBS-0.1\% Tween-20 at room temperature for $1.5 \mathrm{~h}$. The membranes were incubated with primary antibodies against Bcl-2 (cat. no. 4223), Bax (cat. no. 5023) or GAPDH (cat. no. 5174) (all 1:1,000 and purchased from Cell Signaling Technology, Inc.) at $4^{\circ} \mathrm{C}$ overnight. Following the primary incubation, membranes were washed with PBST 3 times and incubated with anti-rabbit IgG horseradish peroxidase-linked antibody (cat. no. 7074; 1:2,000; Cell Signaling Technology, Inc.) at room temperature for $1.5 \mathrm{~h}$. Protein bands were visualized using ECL detection system reagents (MilliporeSigma), according to the manufacturer's instructions. ImageJ v.2.0 software (National Institutes of Health) was used to quantify the band intensity.

Statistical analysis. Statistical analysis was performed using SPSS 20.0 software (IBM Corp.). All experiments were performed in triplicate and data are presented as the mean \pm SD. Unpaired Student's t-test was used to compare differences between two groups, while one-way ANOVA followed by Tukey's post hoc test were used to compare differences between multiple groups. $\mathrm{P}<0.05$ was considered to indicate a statistically significant difference.

\section{Results}

miR-654-3p directly interacts with circRNA_0000285. Previous studies have reported that circRNAs are involved in cancer development by regulating the expression of specific target genes $(8,11)$. Initially, bioinformatics analysis was performed to predict the target gene of circRNA_0000285. miR-654-3p was revealed to be a latent target of circRNA_0000285 (Fig. 1A). Subsequently, the association between circRNA_0000285 and miR-654-3p was confirmed via the dual-luciferase reporter assay (Fig. 1B). Compared with the cells co-transfected with circRNA_0000285 wild-type and mimic control, the luciferase activity of cells co-transfected with circRNA_0000285 wild-type and miR-654-3p mimic were significantly reduced $(\mathrm{P}<0.01$; Fig. $1 \mathrm{~B})$. While no significant changes were observed of the luciferase activity in cells co-transfected with circRNA_0000285 wild-type and mimic control and cells co-transfected with circRNA_0000285 wild-type and miR-654-3p mimic (Fig. 1B). The RNA pull-down assay was performed to confirm the binding between circRNA_0000285 and miR-654-3p, and the pull-down efficiency was verified in TPC-1 and FTC133 cells transfected with circRNA_0000285 or vector (Fig. 1C and D). Compared with Oligo probe, circRNA_0000285 probe significantly enhanced circRNA_0000285 level in TPC-1 and FTC133 cells (all $\mathrm{P}<0.01$; Fig. $1 \mathrm{C}$ and D). miR-654-3p pulled down by biotinylated probes were purified and analyzed via RT-qPCR analysis. The results demonstrated that miR-654-3p was notably pulled down by circRNA_0000285 probe in both TPC-1 and FTC133 cells (Fig. 1E). The results of the RIP assay (Fig. 1F and G) verified that circRNA_0000285 can directly target miR-654-3p, evidenced by significant enhancement of circRNA_0000285 and miR-654-3p in the Anti-Ago2 group in TPC-1 and FTC133 cells compared with in the (all $\mathrm{P}<0.01$; Fig. $1 F$ and $G$ ). Taken together, these results suggest that circRNA_0000285 is a direct target of miR-654-3p.

circRNA_0000285 expression is upregulated and miR-654-3p expression is downregulated in thyroid carcinoma cells. The expression levels of circRNA_0000285 and miR-654-3p in thyroid cancer cell lines were detected via RT-qPCR analysis. circRNA_0000285 expression was significantly upregulated $(\mathrm{P}<0.01$; Fig. $2 \mathrm{~A})$ and miR-654-3p expression was significantly downregulated $(\mathrm{P}<0.01$; Fig. 2B) in the thyroid cancer cell lines (TPC-1 and FTC133) compared with Nthy-ori 3-1 cells.

circRNA_0000285negativelyregulatesmiR-654-3pexpression in thyroid cancer cells. To verify the role of circRNA_0000285 in thyroid cancer, control-siRNA, circRNA_0000285-siRNA, inhibitor control and miR-654-3p inhibitor sequences were transfected into TPC-1 and FTC133 cells for $48 \mathrm{~h}$. RT-qPCR analysis demonstrated that circRNA_0000285 expression significantly decreased in TPC-1 and FTC133 cells following transfection with circRNA_0000285-siRNA compared with the control-siRNA group $(\mathrm{P}<0.01$; Fig. $3 \mathrm{~A}$ and $\mathrm{B})$. In addition, miR-654-3p expression significantly decreased in TPC-1 and 
A

$\begin{array}{rr}\text { WT circ_0000285 } & \text { 5'-GGUGAUGCUUUUCAGCAGACAUU-3' } \\ \text { miR-654-3p } & \text { 3'-UUCCACUACCAGUC---GUCUGUAU-5' }\end{array}$

MUT circ_0000285 5'-GGUGAUGCUUUUCAGGUGUGUAU-3'
$\mathrm{B}$

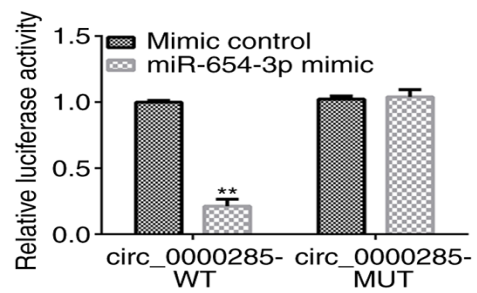

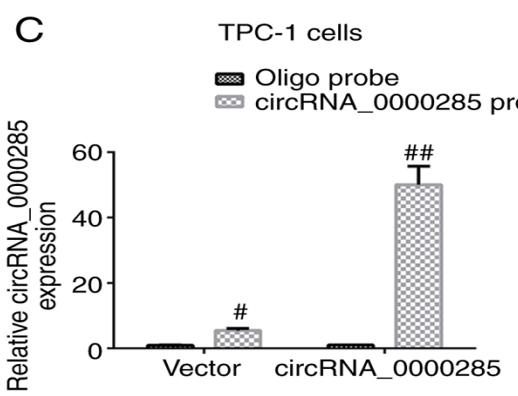

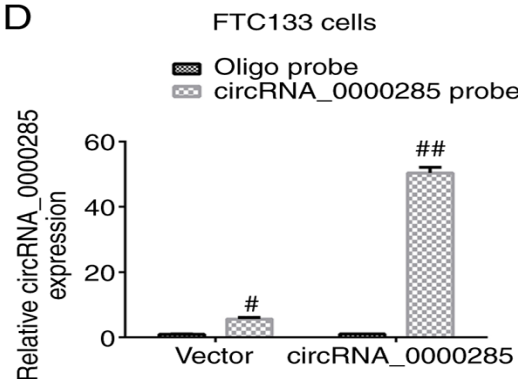

E

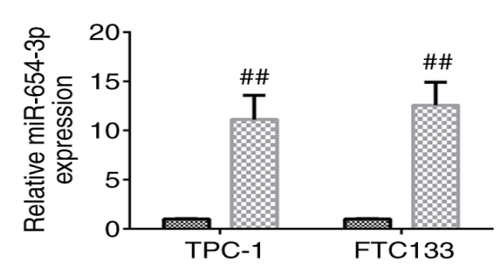

$\mathrm{F}$
TPC-1 cells Anti-IgG $\infty$ Anti-Ago2

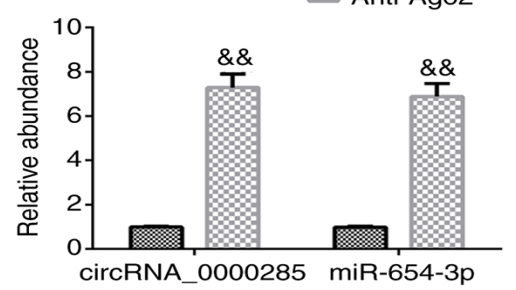

G

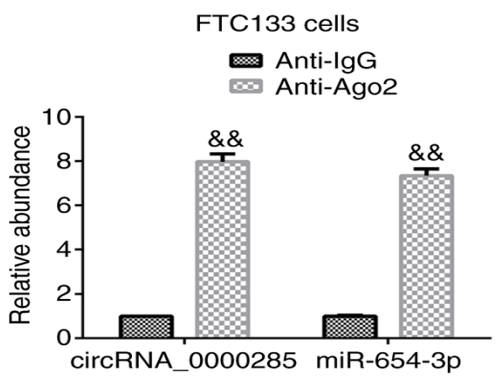

Figure 1. miR-654-3p is a direct target of circRNA_0000285. (A) Schematic diagram of the miR-654-3p binding site in the 3'-untranslated region of circRNA_0000285. (B) The association between miR-654-3p and circRNA_0000285 was verified via the dual-luciferase reporter assay. Relative circRNA_0000285 expression in (C) TPC-1 and (D) FTC133 lysates pulled down by circRNA_0000285 probe or oligo probe. (E) Relative miR-654-3p expression in TPC-1 and FTC133 lysates pulled down by circRNA_0000285 probe or oligo probe. (F and G) The RNA immunoprecipitation assay was performed using Ago2 and IgG antibodies to immunoprecipitate. The expression levels of circRNA_0000285 and miR-654-3p in TPC-1 and FTC133 cells were detected via reverse transcription-quantitative $\mathrm{PCR}$ analysis. ${ }^{* *} \mathrm{P}<0.01$ vs. mimic control; ${ }^{*} \mathrm{P}<0.05$ vs. Oligo probe; ${ }^{* \#} \mathrm{P}<0.01$ vs. Oligo probe; ${ }^{\&} \mathrm{P}<0.01 \mathrm{vs}$. Anti-IgG. miR, microRNA; circRNA, circular RNA; WT, wild-type; MUT, mutant.

A

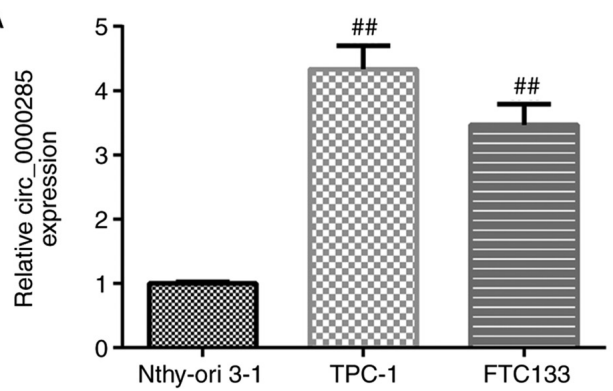

B

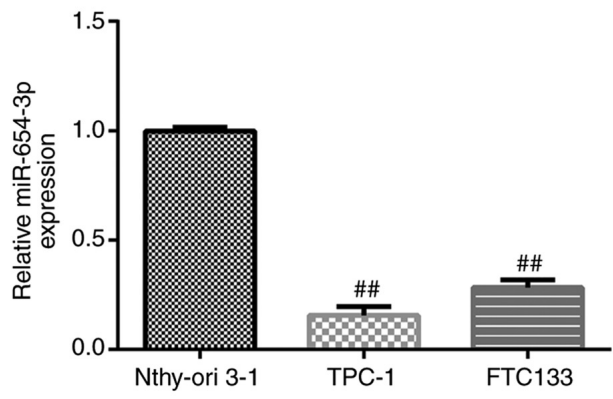

Figure 2. Expression levels of circRNA_0000285 and miR-654-3p in thyroid carcinoma cells. (A) RT-qPCR analysis was performed to detect circRNA_0000285 expression in thyroid cancer cells. (B) RT-qPCR analysis was performed to detect miR-654-3p expression in thyroid cancer cells. ${ }^{\# \#} \mathrm{P}<0.01$ vs. Nthy-ori 3-1 cells. circRNA, circular RNA; miR, microRNA; RT-qPCR, reverse transcription-quantitative PCR.
FTC133 cells transfected with miR-654-3p inhibitor compared with the inhibitor control group $(\mathrm{P}<0.01$; Fig. $3 \mathrm{C}$ and $\mathrm{D})$. Notably, miR-654-3p expression significantly increased following transfection with circRNA_0000285-siRNA, the effect of which was reversed following addition of miR-654-3p inhibitor $(\mathrm{P}<0.01$; Fig. $3 \mathrm{E}$ and $\mathrm{F})$. Collectively, these results suggest that miR-654-3p interferes with circRNA_0000285 expression in thyroid cancer cells.

circRNA_0000285-siRNA inhibits cell proliferation and induces apoptosis by increasing miR-654-3p expression in TPC-1 cells. To further assess the underlying molecular mechanism between miR-654-3p and circRNA_0000285, control-siRNA, circRNA_0000285-siRNA, inhibitor control and miR-654-3p inhibitor sequences were transfected into TPC-1 cells for $48 \mathrm{~h}$ to determine the effects of circRNA 0000285-siRNA on cell proliferation and apoptosis. The results from the MTT assay and flow cytometric analysis demonstrated that circRNA_0000285-siRNA significantly inhibited TPC-1 cell proliferation and promoted apoptosis (all $\mathrm{P}<0.01$; Fig. 4A-C). The expression levels of the apoptosis-associated proteins, Bax and Bcl-2, were detected via western blot analysis. The results demonstrated that transfection with circRNA_0000285-siRNA significantly increased Bax expression and decreased Bcl-2 expression $(\mathrm{P}<0.01$; 
A
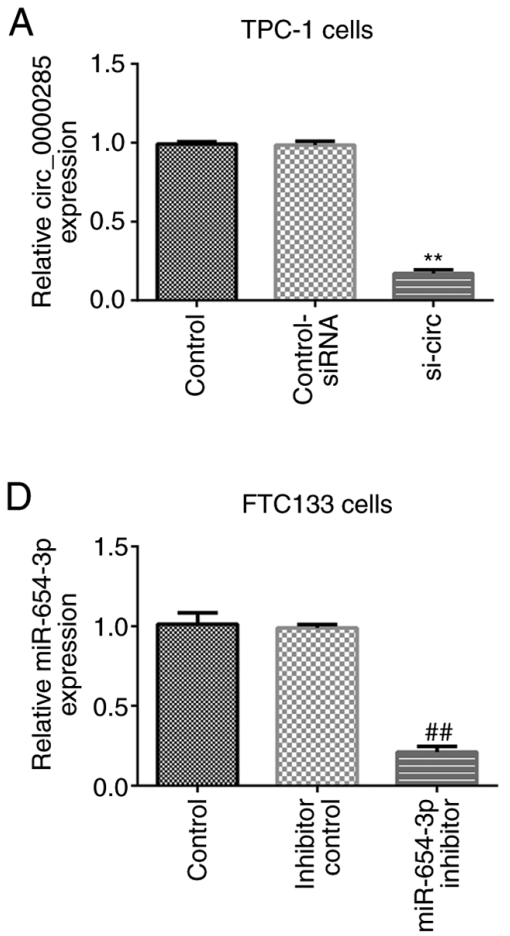

B

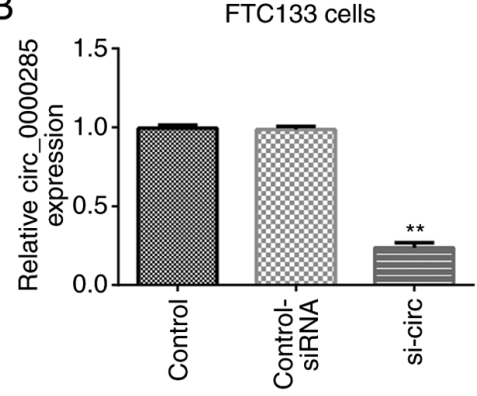

E

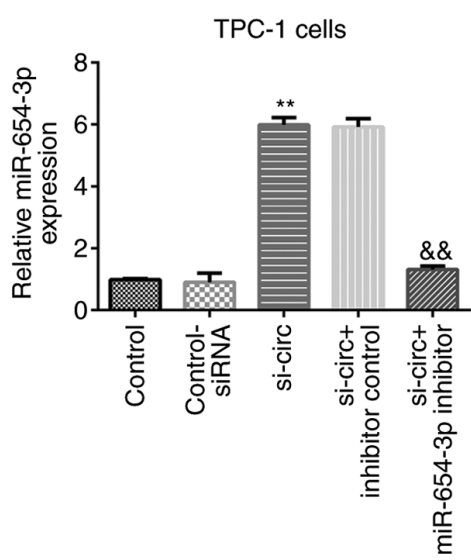

C

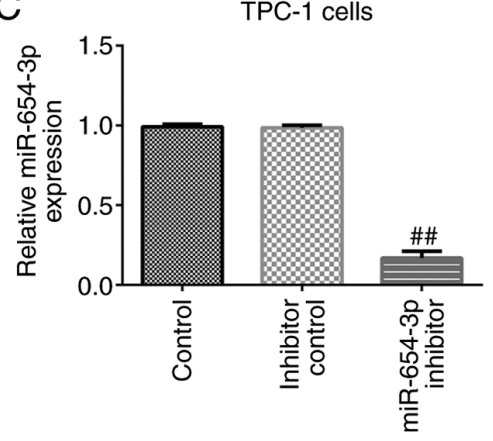

F

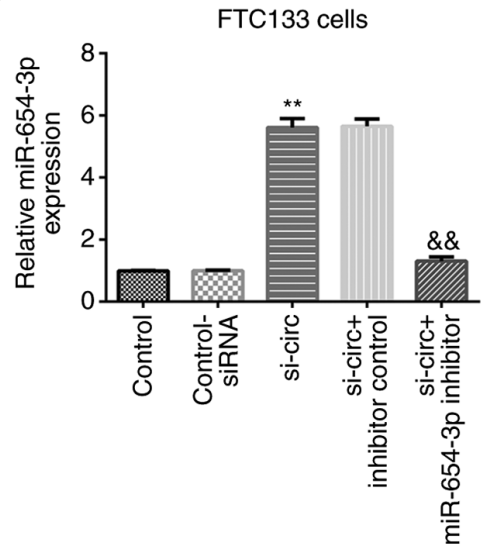

Figure 3. miR-654-3p inhibitor reverses the effects of circRNA_0000285 on miR-654-3p expression. Control-siRNA, circRNA_0000285-siRNA, inhibitor control and miR-654-3p inhibitor sequences were transfected into TPC-1 and FTC133 cells for $48 \mathrm{~h}$. circRNA_0000285 expression was detected via RT-qPCR analysis in (A) TPC-1 and (B) FTC133 cells following transfection with circRNA_0000285-siRNA or control-siRNA. miR-654-3p expression was detected via RT-qPCR analysis in (C) TPC-1 and (D) FTC133 cells following transfection with miR-654-3p inhibitor or inhibitor control. RT-qPCR analysis was performed to detect miR-654-3p expression in (E) TPC-1 and (F) FTC133 cells transfected with circRNA 0000285-siRNA + inhibitor control or circRNA 0000285-siRNA + miR-654-3p inhibitor. ${ }^{* *} \mathrm{P}<0.01$ vs. control-siRNA; ${ }^{\# \#} \mathrm{P}<0.01$ vs. inhibitor control; \& ${ }^{\&} \mathrm{P}<0.01$ vs. si-circ + inhibitor control. miR, microRNA; circRNA, circular RNA; RT-qPCR, reverse transcription-quantitative PCR; si, small interfering.

Fig. 4D-F), and the ratio of Bax/Bcl-2 significantly increased compared with the control-siRNA group $(\mathrm{P}<0.01$; Fig. $4 \mathrm{G})$. Notably, these effects were reversed following transfection with circRNA_0000285-siRNA + miR-654-3p inhibitor (Fig. 4).

circRNA_0000285-siRNA inhibits cell proliferation and induces apoptosis by increasing miR-654-3p expression in FTC133 cells. The effects of circRNA_0000285-siRNA were assessed on FTC133 cell proliferation and apoptosis. Control-siRNA, circRNA_0000285-siRNA, inhibitor control and miR-654-3p inhibitor sequences were transfected into FTC133 cells for $48 \mathrm{~h}$. Transfection with circRNA_0000285-siRNA significantly decreased TPC-1 cell proliferation and promoted induction of TPC-1 cell apoptosis $(\mathrm{P}<0.01$; Fig. $5 \mathrm{~A}-\mathrm{C})$. Western blot analysis demonstrated that circRNA_0000285-siRNA significantly enhanced Bax expression $(\mathrm{P}<0.01$; Fig. 5D and E) and decreased Bcl-2 expression (all $\mathrm{P}<0.01$; Fig. 5D and F), and significantly increased the ratio of Bax/Bcl-2 compared with the control-siRNA group $(\mathrm{P}<0.01$; Fig. 5G). Notably, these effects were reversed following transfection with circRNA_0000285-siRNA + miR-654-3p inhibitor. Taken together, these results suggest that circRNA 0000285-siRNA suppresses cell proliferation and induces apoptosis in thyroid cancer cells by targeting miR-654-3p.

\section{Discussion}

Thyroid cancer is an endocrine tumor with high morbidity and incidence rates (22) and 255,490 incident cases and 41,240 deaths were reported in 2017 worldwide (23). Over the past decade, increasing numbers of thyroid cancer cases have been reported (24). According to previous report, it is estimated that $>64,300$ newly diagnosed cases of thyroid cancer will emerge in the United States in 2016 (25). Thus, effective therapeutic methods are required to reduce the burden of patients with thyroid cancer.

circRNAs are a type of non-coding RNAs that play important roles in oncogenesis, including thyroid cancer development $(26,27)$. Increasing evidence suggest that circRNAs are involved in cellular processes, such as cell proliferation, apoptosis, differentiation and invasion, and that they may be used as novel biomarkers or therapeutic targets for disease treatment. For example, Zhang et al (28) confirmed that circ_0067934 depletion accelerates cell apoptosis and represses cell proliferation, migration and invasion in thyroid cancer by sponging miR-1304 and regulating C-X-CR motif chemokine receptor 1 expression. hsa_circ_0000285 is a novel circRNA that is dysregulated in various cancers and disease types, including osteosarcoma (29), diabetic nephropathy (30) and laryngocarcinoma (31). However, the specific role and molecular mechanism of circ_0000285 in thyroid cancer 
A
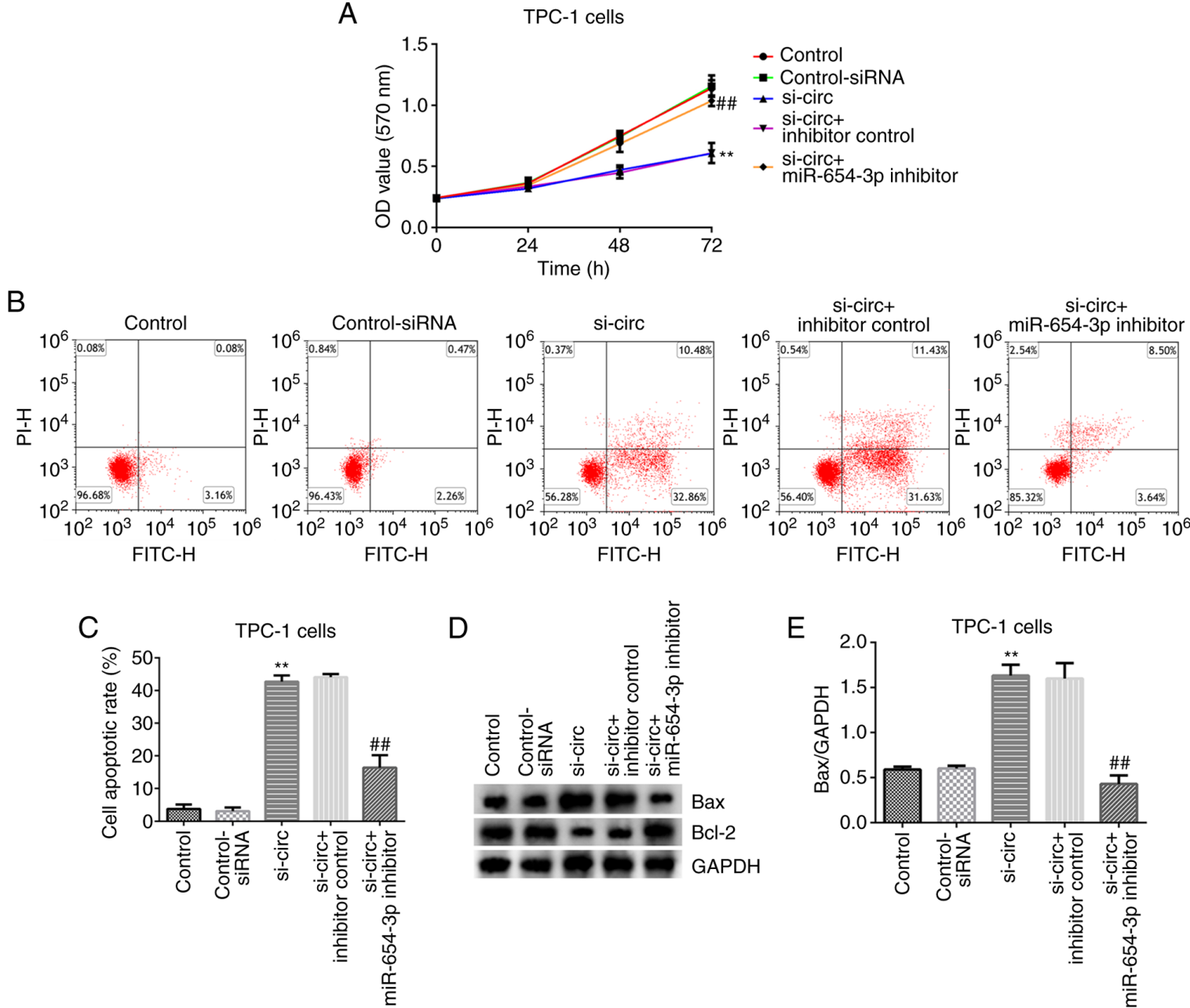

D
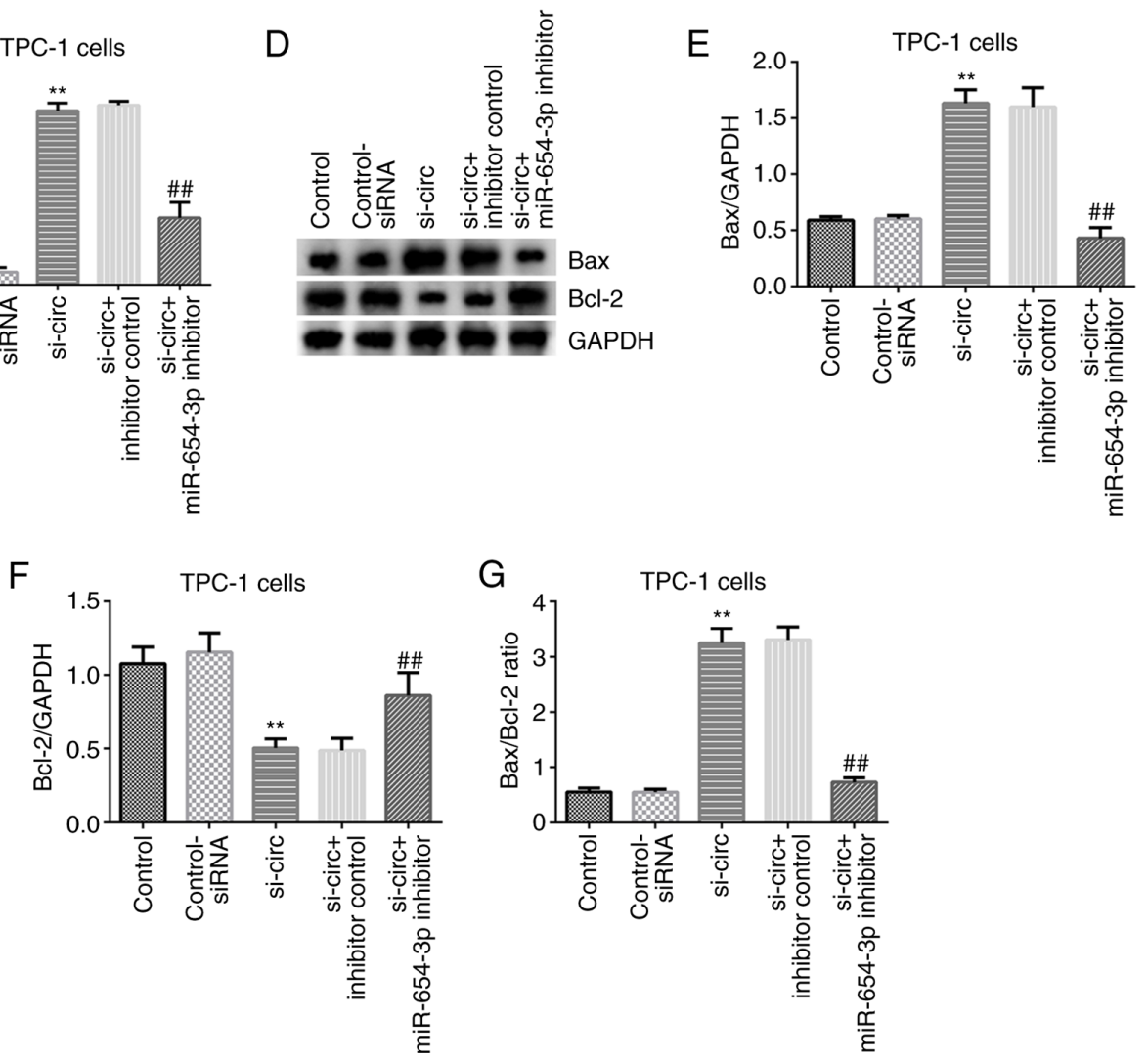

Figure 4. miR-654-3p inhibitor reverses the effects of circRNA_0000285 on TPC-1 cell proliferation and apoptosis. Control-siRNA, circRNA_0000285-siRNA, inhibitor control and miR-654-3p inhibitor sequences were transfected into TPC-1 cells for $48 \mathrm{~h}$. (A) The viability of TPC-1 cells was assessed via the MTT assay. (B) Detection of apoptotic TPC-1 cells was assessed via flow cytometric analysis. (C) Quantitative analysis of apoptotic cells. (D) Western blot analysis was performed to detect the protein expression levels of Bax and Bcl-2 in the different groups. (E) Bax/GAPDH ratio and (F) Bcl-2/GAPDH ratio. (G) Assessment of the Bax/Bcl-2 ratio. ${ }^{* *} \mathrm{P}<0.01$ vs. control-siRNA; ${ }^{\# \#} \mathrm{P}<0.01$ vs. si-circ + inhibitor control. miR, microRNA; circRNA, circular RNA; si, small interfering; Bcl-2, B-cell lymphoma 2; OD, optical density; PI, propidium iodide.

have not yet been fully investigated. Thus, the present study investigated the underlying molecular mechanism of circRNA_0000285 in thyroid cancer to identify novel treatment methods for this disease.

A previous study revealed the interaction sites between circRNA_0000285 and miR-654-3p (18). However, the association between circRNA_0000285 and miR-654-3p in thyroid cancer remain unknow. To the best of our knowledge, the present study was the first to investigate the association between circRNA_0000285 and miR-654-3p in thyroid cancer. The present study confirmed the binding between circRNA_0000285 and miR-654-3p via the dual-luciferase reporter and RIP assays. The results revealed that miR-654-3p directly interacted with circRNA_0000285. The present study also assessed the role of circRNA_0000285 in thyroid cancer cells. Its expression was detected, along with 
A
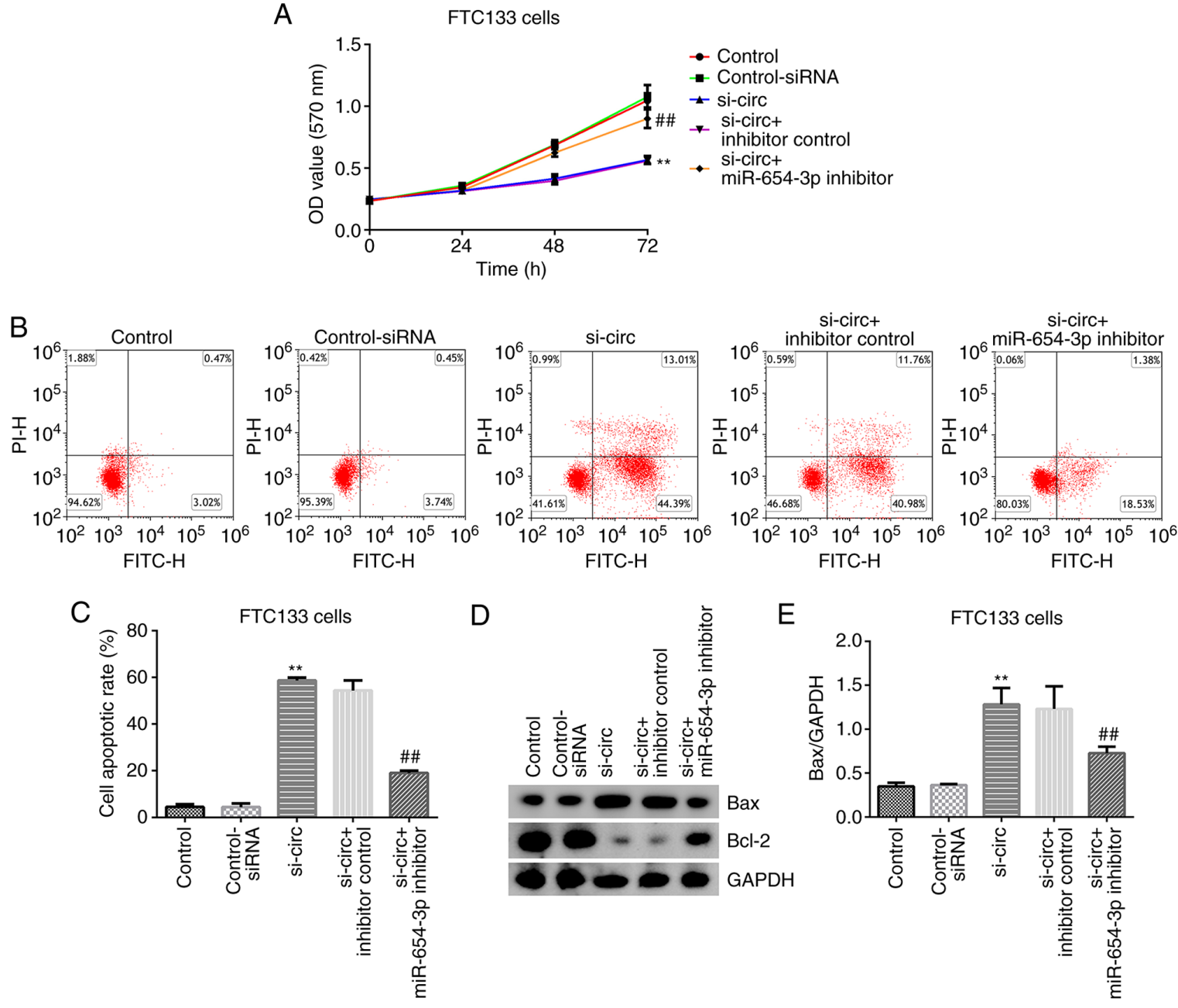

D
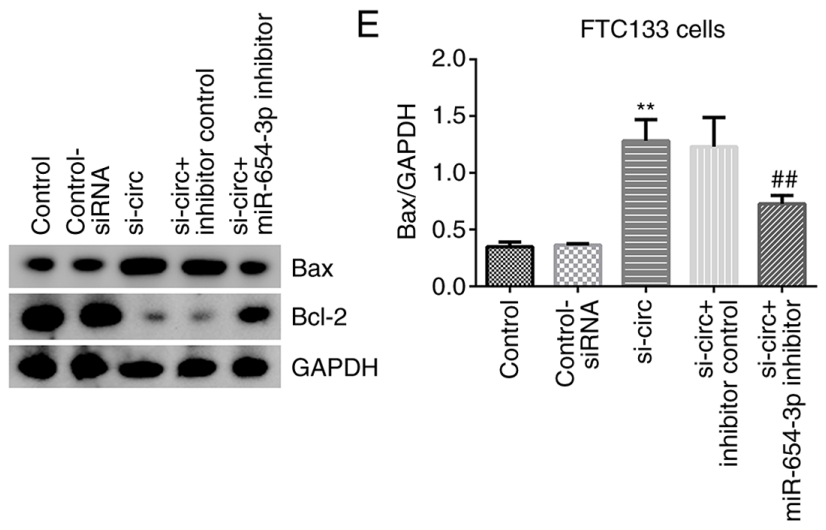

$\mathrm{F}$

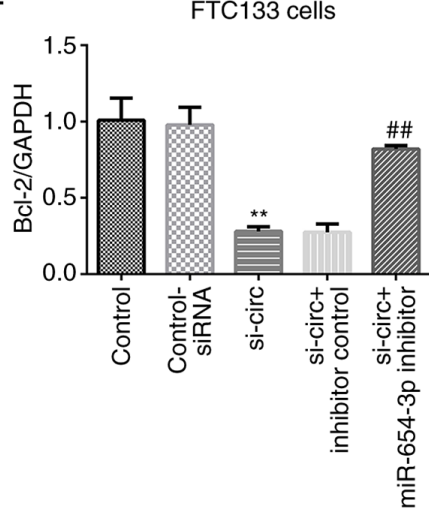

G

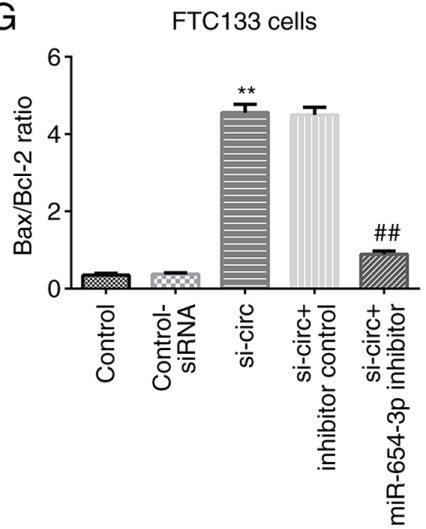

Figure 5. miR-654-3p inhibitor reverses the effects of circRNA_0000285 on FTC133 cell viability and apoptosis. Control-siRNA, circRNA_0000285-siRNA, inhibitor control and miR-654-3p inhibitor sequences were transfected into FTC133 cells for 48 h. (A) The viability of FTC133 cells was assessed via the MTT assay. (B) Detection of apoptotic FTC133 cells was assessed via flow cytometric analysis. (C) Quantitative analysis of apoptotic cells. (D) Western blot analysis was performed to detect the protein expression levels of Bax and Bcl-2 levels in the different groups. (E) Bax/GAPDH ratio and (F) Bcl-2/GAPDH ratio. (G) Assessment of the Bax/Bcl-2 ratio. ${ }^{* *} \mathrm{P}<0.01$ vs. control-siRNA; ${ }^{\#} \mathrm{P}<0.01$ vs. si-circ + inhibitor control. miR, microRNA; circRNA, circular RNA; si, small interfering; Bcl-2, B-cell lymphoma 2; OD, optical density; PI, propidium iodide.

miR-654-3p expression, in the thyroid cancer cell lines, TPC-1 and FTC133, and Nthy-oir 3-1 cells. The results demonstrated that circRNA_0000285 expression was upregulated, whereas miR-654-3p expression was downregulated in thyroid cancer cells compared with Nthy-ori 3-1 cells. Taken together, these results suggest that circ_0000285 functions as an oncogene in thyroid tumors by regulating miR-654-3p expression.
Previous studies have reported that abnormal regulation of miRNAs is associated with the development and aggressiveness of several tumors (32-34). Altered expression levels of circRNA_0000285 or miR-654-3p may affect the functions of thyroid cancer cells. Control-siRNA, circRNA_0000285-siRNA, inhibitor control and miR-654-3p inhibitor sequences were transfected into TPC-1 and 
FTC133 cells for $48 \mathrm{~h}$. Transfection efficiency was assessed via RT-qPCR analysis. The results demonstrated that circRNA_0000285-siRNA suppressed circRNA_0000285 expression in TPC-1 and FTC133 cells. In addition, transfection with miR-654-3p inhibitor significantly inhibited miR-654-3p expression, whereas circRNA_0000285-siRNA promoted miR-654-3p expression in TPC-1 and FTC133 cells compared with the control-siRNA group. Notably, these results were reversed following transfection with miR-654-3p inhibitor. Collectively, these results suggest that circRNA_0000285 negatively regulates miR-654-3p expression in TPC-1 and FTC133 cells.

Defective apoptosis is a main characteristic of tumor cells, whereby induction of apoptosis may prevent cancer development (35). It has been reported that long non-coding RNA urothelial cancer associated 1 promotes gastric cancer proliferation, migration and inhibits apoptosis by sponging antitumor miRNAs (36). In the present study, transfection of thyroid cancer cells with circRNA_0000285-siRNA decreased cell viability, which was accompanied by increased cell apoptosis in the circRNA_0000285-siRNA group. These effects were reversed following transfection of cells with miR-654-3p inhibitor.

Bax is a pro-apoptotic protein that is a transcriptional target for p53 (37). Bcl-2 can inhibit the activity of Bax and induce cell apoptosis (38). To further investigate the molecular mechanism of circRNA_0000285 and miR-654-3p in thyroid cancer cells, the expression levels of Bax and Bcl-2 were detected in the different groups. The results demonstrated that circRNA_0000285-siRNA enhanced Bax expression, decreased $\mathrm{Bcl}-2$ expression and increased the ratio of Bax/Bcl-2 compared with the control-siRNA group. Notably, these effects were reversed following transfection with circRNA_0000285-siRNA + miR-654-3p inhibitor. Taken together, these results suggest that circRNA_0000285-siRNA inhibits thyroid cancer cell proliferation and induces apoptosis by increasing miR-654-3p expression.

The present study was an in vitro preliminary study of the role of circRNA_0000285 in the proliferation and apoptosis of thyroid cancer cells. To confirm the role of circRNA_0000285 in thyroid cancer, in-depth experiments are required. For example, determining the role of miR-654-3p alone in thyroid cancer cells is required to verify the results presented here. In addition, the specific molecular mechanism of circRNA_0000285/miR-654-3p affecting the viability of thyroid cancer cells requires investigation. Prospective studies are also required to investigate other target genes downstream of circRNA_0000285 or miR-654-3p in thyroid cancer. Furthermore, the role of circRNA_0000285 in thyroid cancer should be determined in vivo.

In conclusion, the results of the present study demonstrated that circRNA_0000285-siRNA repressed thyroid cancer cell proliferation and induced apoptosis by regulating miR-654-3p expression. These findings may provide evidence for a novel biomarker and a potential therapeutic target for thyroid cancer.

\section{Acknowledgements}

Not applicable.

\section{Funding}

No funding was received.

\section{Availability of data and materials}

The datasets used and/or analyzed during the current study are available from the corresponding author upon reasonable request.

\section{Authors' contributions}

RG contributed to the study design, data collection, statistical analysis, data interpretation and manuscript preparation. HY contributed to data collection, statistical analysis and manuscript preparation. QG, NW, YZ and HD contributed to data collection and statistical analysis. RG and HY performed the experiments. RG and HY confirmed the authenticity of all the raw data. All authors read and approved the final manuscript.

\section{Ethics approval and consent to participate}

Not applicable.

\section{Patient consent for publication}

Not applicable.

\section{Competing interests}

The authors declare that they have no competing interests.

\section{References}

1. Peng C, Li Z, Gao H, Zou X, Wang X, Zhou C and Niu J: Synchronous primary sigmoid colon cancer and primary thyroid cancer followed by a malignant tumor of the kidney: Case report of multiple primary cancer and review of the literature. Oncol Lett 17: 2479-2484, 2019.

2. Kaptan E, Sancar-Bas S, Sancakli A, Bektas S and Bolkent S: The effect of plant lectins on the survival and malignant behaviors of thyroid cancer cells. J Cell Biochem 119: 6274-6287, 2018.

3. Chow TL: Quality of life after surgical treatment for thyroid cancer. JAMA Otolaryngol Head Neck Surg 145: 873, 2019.

4. Iniguez-Ariza NM, Stan MN and Bible KC: Effect of thyroid hormone suppression on control of advanced well-differentiated thyroid cancer. Endocrine 59: 228-229, 2018.

5. Barakat S, Odem J, Batanian JR, Raza S and Khan UZ: Papillary thyroid cancer in struma testis with malignant transformation in the lung associated with trisomy 17 successfully treated with total thyroidectomy and radioiodine ablation. Case Rep Oncol 7: 751-757, 2014.

6. Luo X and Wu ACYZYJBZJ: Analysis of risk factors for postoperative recurrence of thyroid cancer. J BUON 24: 813-818, 2019.

7. Lee E, Elhassan S, Lim GPL, Kok WH, Tan SW, Leong EN, Tan SH, Chan EWL, Bhattamisra SK, Rajendran R and Candasamy M: The roles of circular RNAs in human development and diseases. Biomed Pharmacother 111: 198-208, 2019

8. Ma HB, Yao YN, Yu JJ, Chen XX and Li HF: Extensive profiling of circular RNAs and the potential regulatory role of circRNA-000284 in cell proliferation and invasion of cervical cancer via sponging miR-506. Am J Transl Res 10: 592-604, 2018.

9. Panda AC: Circular RNAs act as miRNA sponges. Adv Exp Med Biol 1087: 67-79, 2018

10. Dori $\mathrm{M}$ and Bicciato S: Integration of bioinformatic predictions and experimental data to identify circRNA-miRNA associations. Genes (Basel) 10: 642, 2019. 
11. Zhang W and Zhang S: Down-regulation of circRNA 0000285 suppresses cervical cancer development by regulating miR197-3p-ELK1 Axis. Cancer Manag Res 12: 8663-8674, 2020.

12. Shirjang S, Mansoori B, Asghari S, Duijf P, Mohammadi A, Gjerstorff $M$ and Baradaran B: MicroRNAs in cancer cell death pathways: Apoptosis and necroptosis. Free Radic Biol Med 139: $1-15,2019$.

13. Carroll AP, Goodall GJ and Liu B: Understanding principles of miRNA target recognition and function through integrated biological and bioinformatics approaches. Wiley Interdiscip Rev RNA 5: 361-379, 2014

14. Liu S, Wang H, Mu J, Wang H, Peng Y, Li Q, Mao D and Guo L: miRNA-211 triggers an autophagy-dependent apoptosis in cervical cancer cells: Regulation of Bcl-2. Naunyn Schmiedebergs Arch Pharmacol 393: 359-370, 2020.

15. Geraldo MV, Nakaya HI and Kimura ET: Down-regulation of $14 \mathrm{q} 32$-encoded miRNAs and tumor suppressor role for miR-654-3p in papillary thyroid cancer. Oncotarget 8: 9597-9607, 2017.

16. Yang J, Zhang Z, Chen S, Dou W, Xie R and Gao J: miR-654-3p predicts the prognosis of hepatocellular carcinoma and inhibits the proliferation, migration, and invasion of cancer cells. Cancer Biomark 28: 73-79, 2020

17. Pu JT, Hu Z, Zhang DG, Zhang T, He KM and Dai TY: miR-654-3p suppresses non-small cell lung cancer tumourigenesis by inhibiting PLK4. Onco Targets Ther 13 7997-8008, 2020.

18. Yao T, Zha D, Hu C and Wu X: circ 0000285 promotes podocyte injury through sponging miR-654-3p and activating MAPK6 in diabetic nephropathy. Gene 747: 144661, 2020.

19. Dong W, Bi J, Liu H, Yan D, He Q, Zhou Q, Wang Q, Xie R, Su Y, Yang M, et al: Circular RNA ACVR2A suppresses bladder cancer cells proliferation and metastasis through miR-626/EYA4 axis. Mol Cancer 18: 95, 2019.

20. Bierhoff $\mathrm{H}$ : Analysis of lncRNA-protein interactions by RNA-Protein Pull-Down assays and RNA immunoprecipitation (RIP). Methods Mol Biol 1686: 241-250, 2018.

21. Livak KJ and Schmittgen TD: Analysis of relative gene expression data using real-time quantitative PCR and the 2(-Delta Delta C(T)) method. Methods 25: 402-408, 2001

22. Pajamaki N, Metso S, Hakala T, Ebeling T, Huhtala H, Ryödi E, Sand J, Jukkola-Vuorinen A, Kellokumpu-Lehtinen PL and Jaatinen P: Long-term cardiovascular morbidity and mortality in patients treated for differentiated thyroid cancer. Clin Endocrinol (Oxf) 88: 303-310, 2018.

23. Deng Y, Li H, Wang M, Li N, Tian T, Wu Y, Xu P, Yang S, Zhai Z, Zhou L, et al: Global burden of thyroid cancer from 1990 to 2017 JAMA Netw Open 3: e208759, 2020.

24. Burton BN, Okwuegbuna O, Jafari A, Califano J, Brumund KT and Gabriel RA: Association of preoperative anemia with 30-day morbidity and mortality among patients with thyroid cancer who undergo thyroidectomy. JAMA Otolaryngol Head Neck Surg 145: 124-1231, 2019

25. Mao Y and Xing M: Recent incidences and differential trends of thyroid cancer in the USA. Endocr Relat Cancer 23: 313-322, 2016.
26. Wu G, Zhou W, Pan X, Sun Z, Sun Y, Xu H, Shi P, Li J, Gao L and Tian X: Circular RNA profiling reveals exosomal circ_0006156 as a novel biomarker in papillary thyroid cancer. Mol Ther Nucleic Acids 19: 1134-1144, 2020.

27. Cui W and Xue J: Circular RNA DOCK1 down-regulates microRNA-124 to induce the growth of human thyroid cancer cell lines. Biofactors 46: 591-599, 2020.

28. Zhang H, Ma XP, Li X and Deng FS: Circular RNA circ 0067934 exhaustion expedites cell apoptosis and represses cell proliferation, migration and invasion in thyroid cancer via sponging miR-1304 and regulating CXCR1 expression. Eur Rev Med Pharmacol Sci 23: 10851-10866, 2019.

29. Long Z, Gong F, Li Y, Fan Z and Li J: circ 0000285 regulates proliferation, migration, invasion and apoptosis of osteosarcoma by miR-409-3p/IGFBP3 axis. Cancer Cell Int 20: 481, 2020.

30. Yao T, Zha D, Hu C and Wu X: circ 0000285 promotes podocyte injury through sponging miR-654-3p and activating MAPK6 in diabetic nephropathy. Gene 747: 144661, 2020.

31. Qin JB, Chang W, Yuan GH, Huang L and Qiu ZF: Circular RNA hsa circ 0000285 acts as an oncogene in laryngocarcinoma by inducing Wnt/beta-catenin signaling pathway. Eur Rev Med Pharmacol Sci 24: 9773, 2020.

32. Bao B, Ali S, Ahmad A,Li Y,Banerjee S, Kong D, Aboukameel A, Mohammad R, Van Buren E, Azmi AS and Sarkar FH: Differentially expressed miRNAs in cancer-stem-like cells: Markers for tumor cell aggressiveness of pancreatic cancer. Stem Cells Dev 23: 1947-1958, 2014.

33. Buruiană A, Florian SI, Florian AI, Timis TL, Mihu CM, Miclăuș M, Oșan S, H̉rapșa I, Cataniciu RC, Farcaș M and Susman S: The roles of miRNA in glioblastoma tumor cell communication: Diplomatic and aggressive negotiations. Int J Mol Sci 21: 1950, 2020.

34. Donnarumma E, Fiore D, Nappa M, Roscigno G, Adamo A Iaboni M, Russo V, Affinito A, Puoti I, Quintavalle C, et al: Cancer-associated fibroblasts release exosomal microRNAs that dictate an aggressive phenotype in breast cancer. Oncotarget 8: 19592-19608, 2017.

35. Zhao Y,Ponnusamy M, Dong Y,Zhang L, Wang K and Li P: Effects of miRNAs on myocardial apoptosis by modulating mitochondria related proteins. Clin Exp Pharmacol Physiol 44: 431-440, 2017.

36. Wang CJ, Zhu CC, Xu J, Wang M, Zhao WY, Liu Q, Zhao G and Zhang ZZ: The lncRNA UCA1 promotes proliferation, migration, immune escape and inhibits apoptosis in gastric cancer by sponging anti-tumor miRNAs. Mol Cancer 18: 115, 2019.

37. Lee SB, Lee S, Park JY, Lee SY and Kim HS: Induction of p53-dependent apoptosis by prostaglandin $\mathrm{A}_{2}$. Biomolecules 10 : $492,2020$.

38. Azimian H, Dayyani M, Toossi M and Mahmoudi M: Bax/Bcl-2 expression ratio in prediction of response to breast cancer radiotherapy. Iran J Basic Med Sci 21: 325-332, 2018.

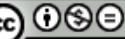

This work is licensed under a Creative Commons Attribution-NonCommercial-NoDerivatives 4.0 International (CC BY-NC-ND 4.0) License. 